\title{
Special Subgroups of Regular Semigroups
}

\section{T. S. Blyth \& M. H. Almeida Santos}

To cite this article: T. S. Blyth \& M. H. Almeida Santos (2016): Special Subgroups of Regular Semigroups, Communications in Algebra, DOI: 10.1080/00927872.2016.1262385

To link to this article: http://dx.doi.org/10.1080/00927872.2016.1262385

Accepted author version posted online: 01 Dec 2016.

Submit your article to this journal $\sqrt{2}$

Q View related articles ¿

View Crossmark data \lceil 


\title{
Special subgroups of regular semigroups
}

\author{
T. S. Blyth ${ }^{1}$ and M. H. Almeida Santos ${ }^{2}$ \\ ${ }^{1}$ Mathematical Institute, University of St Andrews, Scotland \\ ${ }^{2}$ Centro de Matemática e Aplicações (CMA), Departamento de Matemática, FCT, Universidade
}

Nova de Lisboa, Portugal

\begin{abstract}
Extending the notions of inverse transversal and associate subgroup, we consider a regular semigroup $S$ with the property that there exists a subsemigroup $T$ which contains, for each $x \in S$, a unique $y$ such that both $x y$ and $y x$ are idempotent. Such a subsemigroup is necessarily a group which we call a special subgroup. Here we investigate regular semigroups with this property. In particular, we determine when the subset of perfect elements is a subsemigroup and describe its structure in naturally arising situations.
\end{abstract}

KEYWORDS: Quasi-ideal; regular semigroup; special subgroup

2010 Mathematics Subject Classification: 20M20; 20M17

Received 6 April 2016

T. S. Blyth tsb@st-andrews.ac.uk Mathematical Institute, University of St Andrews, Scotland. 


\section{SPECIAL SUBGROUPS}

If $S$ is a regular semigroup then for each $x \in S$ we denote the set of inverses of $x$ by $V(x)$, and the set of associates of $x$ by $A(x)=\{y \in S \mid x y x=x\}$. Well known is the concept of an inverse transversal of $S$, namely an inverse subsemigroup $S^{\circ}=\left\{x^{\circ} \mid x \in S\right\}$ with the property that $\left|S^{\circ} \cap V(x)\right|=1$ for every $x \in S$ (1). Corresponding to this is the notion of an associate subgroup of $S$, namely a subgroup $S^{\star}=\left\{x^{\star} \mid x \in S\right\}$ with the property that $\left|S^{\star} \cap A(x)\right|=1$ for every $x \in S(2)$. Here we shall be concerned with the subset

$I(x)=\{y \in S \mid x y, y x \in E(S)\}$

where $E(S)$ denotes the set of idempotents of $S$. Clearly, $V(x) \subseteq A(x) \subseteq I(x)$. Moreover, equality holds throughout if and only if $S$ is completely simple (5)(6).

Our purpose here is to investigate the more general notion of a subsemigroup $T$ of $S$ with the property that

$(\forall x \in S) \quad|T \cap I(x)|=1$.

Given such a subsemigroup $T$, we define $x^{\sim}$ for every $x \in S$ by

$T \cap I(x)=\left\{x^{\sim}\right\}$

Thus $x x^{\sim}, x^{\sim} x \in E(S)$ for every $x \in S$, and we observe first that every $x \in T$ is such that $x \in$ $T \cap I\left(x^{\sim}\right)=\left\{x^{\sim \sim}\right\}$ whence $x=x^{\sim \sim}$ and consequently $T \subseteq S^{\sim}=\left\{x^{\sim} \mid x \in S\right\}$. The converse 
inclusion being clear from the definition of $x^{\sim}$, it follows that $T=S^{\sim}$. Then $x^{\sim}=x^{\sim \sim \sim}$ for every $x \in S$. Since $T$ is a subsemigroup, for every $x \in T$ we have $x^{\sim} x x^{\sim} \in T \cap I(x)$ whence $x^{\sim} x x^{\sim}=x^{\sim}$ and so $x x^{\sim} x \in V\left(x^{\sim}\right) \subseteq I\left(x^{\sim}\right)$. Since also $x x^{\sim} x \in T$ it follows that every $x \in T$ is such that $x x^{\sim} x=x^{\sim \sim}=x$ and so $S^{\sim}=T$ is regular. Now if $y$ is any inverse of $x^{\sim}$ in $S^{\sim}$ then $y \in S^{\sim} \cap I\left(x^{\sim}\right)=\left\{x^{\sim \sim}\right\}$ whence $y=x^{\sim \sim}$ and consequently $S^{\sim}$ is an inverse semigroup in which $\left(x^{\sim}\right)^{-1}=x^{\sim \sim}$. But if $e, f \in E\left(S^{\sim}\right)$ then $e f=f e \in E\left(S^{\sim}\right)$ gives $e, f \in S^{\sim} \cap I(e f)=\left\{(e f)^{\sim}\right\}$ whence $e=(e f)^{\sim}=f$. Thus we conclude that $S^{\sim}$ is a subgroup of $S$.

In what follows we shall call such a subgroup $S^{\sim}$ a special subgroup of $S$.

Theorem 1. Let $S^{\sim}$ be a special subgroup of the regular semigroup $S$. If $\xi$ is the identity element of $S^{\sim}$ then $S^{\sim}=H_{\xi}$.

Proof. Since the maximal subgroups of $S$ are precisely the $\mathcal{H}$-classes which contain idempotents, it follows that $S^{\sim} \subseteq H_{\xi}$. To obtain the reverse inclusion, let $x \in H_{\xi}$. Then $x x^{\sim}, x^{\sim} x \in E\left(H_{\xi}\right)$ and so $x x^{\sim}=\xi=x^{\sim} x$, whence $x^{-1}=x^{\sim}$ and $x=\left(x^{\sim}\right)^{-1}=x^{\sim \sim} \in S^{\sim}$. Thus $S^{\sim}=H_{\xi}$.

Example 1. Let $S$ be an orthodox completely simple semigroup. As is well-known, we can represent $S$ as the cartesian product semigroup $S=G \times B$ of a group $G$ and a rectangular band $B$. Choose and fix an element $\alpha \in B$. The $\mathcal{H}$-class of the idempotent $e=\left(1_{G}, \alpha\right)$, namely $H_{e}=G \times\{\alpha\}$, is a group transversal of $S$ under the definition $(g, x)^{\circ}=\left(g^{-1}, \alpha\right)$. Let $S^{1}$ be the 
monoid obtained from $S$ by the adjunction of a new identity element 1 and define, for each $t \in S^{1}$, $t^{\sim}= \begin{cases}t^{\circ} & \text { if } t \in S \\ e & \text { if } t=1\end{cases}$

Then $\left(S^{1}\right)^{\sim}=H_{e}$. For each $(g, x) \in S$ we have that

$(h, y) \in H_{e} \cap I(g, x) \Longleftrightarrow h=g^{-1}, y=\alpha$

whence $H_{e} \cap I(g, x)=\left\{\left(g^{-1}, \alpha\right)\right\}=\left\{(g, x)^{\circ}\right\}=\left\{(g, x)^{\sim}\right\}$. Since also $H_{e} \cap I(1)=H_{e} \cap E\left(S^{1}\right)=$ $\{e\}=\left\{1^{\sim}\right\}$, it follows that $H_{e}$ is a special subgroup of $S^{1}$. Note that since $A(1)=\{1\}$ the semigroup $S^{1}$ has no associate subgroups. Indeed, any such subgroup would have to contain 1 and the only subgroup of $S^{1}$ which does so is $\{1\}$.

Example 2. Let $k>1$ be a fixed integer and for each $n \in \mathbb{Z}$ let $n_{k}$ be the biggest multiple of $k$ that is less than or equal to $n$. Consider the cartesian product set $S=\mathbb{Z} \times \mathbb{Z}$ equipped with the multiplication

$(m, n)(p, q)=\left(\max \{m, p\}, n+q_{k}\right)$.

Since $m_{k}+n_{k}=\left(m+n_{k}\right)_{k}=\left(m_{k}+n\right)_{k}$ it follows that $S$ is a semigroup which is regular; for example, $(m, n)\left(m,-n_{k}\right)(m, n)=(m, n)$. Here $E(S)=\left\{(m, n) \mid n_{k}=0\right\}$ and

$I(m, n)=\left\{(p, q) \mid q_{k}+n_{k}=0\right\}=\left\{(p, q) \mid-n_{k} \leqslant q \leqslant-n_{k}+k-1\right\}$. 
The subsemigroup $S^{\sim}=\left\{\left(0, t_{k}\right) \mid t \in \mathbb{Z}\right\}$ is such that $S^{\sim} \cap I(m, n)=\left\{\left(0,-n_{k}\right)\right\}$ and so is a special subgroup. Here also

$V(m, n)=\left\{(p, q) \mid p=m, q_{k}+n_{k}=0\right\} ; \quad A(m, n)=\left\{(p, q) \mid p \leqslant m, q_{k}+n_{k}=0\right\}$.

The following result, which involves formulae similar to those for inverse transversals, will be used throughout what follows.

Theorem 2. Let $S$ be a regular semigroup. If $S^{\sim}$ is a special subgroup of $S$ then

(1) $(\forall x, y \in S) \quad\left(x y^{\sim}\right)^{\sim}=y^{\sim \sim} x^{\sim}$ and $\left(x^{\sim} y\right)^{\sim}=y^{\sim} x^{\sim \sim}$;

(2) $(\forall x, y \in S) \quad(x y)^{\sim}=\left(x^{\sim} x y\right)^{\sim} x^{\sim}=y^{\sim}(x y y \sim)^{\sim}$.

Proof.

(1) Let $\xi$ be the identity element of $S^{\sim}$. Then on the one hand $x y^{\sim} \cdot y^{\sim \sim} x^{\sim}=x \xi x^{\sim}=x x^{\sim} \in E(S)$, and on the other,

$y^{\sim \sim} x^{\sim} x y^{\sim} \cdot y^{\sim \sim} x^{\sim} x y^{\sim}=y^{\sim \sim} x^{\sim} x \xi x^{\sim} x y^{\sim}=y^{\sim \sim} x^{\sim} x y^{\sim}$

whence also $y^{\sim \sim} x^{\sim} \cdot x y^{\sim} \in E(S)$. Consequently, $y^{\sim \sim} x^{\sim} \in S^{\sim} \cap I\left(x y^{\sim}\right)$ and hence $\left(x y^{\sim}\right)^{\sim}=y^{\sim \sim} x^{\sim}$.

Similarly, $\left(x^{\sim} y\right)^{\sim}=y^{\sim} x^{\sim \sim}$. 
(2) Using (1), we have $\left(x^{\sim} x y\right)^{\sim} x^{\sim}=(x y)^{\sim} x^{\sim \sim} x^{\sim}=(x y)^{\sim} \xi=(x y)^{\sim}$, and similarly $y^{\sim}\left(x y y^{\sim}\right)^{\sim}=$ $y^{\sim} y^{\sim \sim}(x y)^{\sim}=\xi(x y)^{\sim}=(x y)^{\sim}$.

\section{PARTICULAR SUBSETS}

In the presence of an inverse transversal $S^{\circ}$, or of an associate subgroup $S^{\star}$, Green's relations are nicely describable. Indeed, in those situations they are as follows:

$$
\begin{array}{ll}
(x, y) \in \mathcal{R} \Longleftrightarrow x x^{\circ}=y y^{\circ} & {\left[\text { resp. } x x^{\star}=y y^{\star}\right]} \\
(x, y) \in \mathcal{L} \Longleftrightarrow x^{\circ} x=y^{\circ} y & {\left[\text { resp. } x^{\star} x=y^{\star} y\right] .}
\end{array}
$$

This is not so in general for regular semigroups with special subgroups. For instance, in Example 1 we have $e e^{\sim}=e=11^{\sim}$ but $(e, 1) \notin \mathcal{R}$ since $1 \notin e S^{1}$. So a separate investigation of the sets $J=\left\{x x^{\sim} \mid x \in S\right\}$ and $K=\left\{x^{\sim} x \mid x \in S\right\}$ is warranted. For this, we note that $J$ and $K$ have the equivalent descriptions

$J=\left\{x \in S \mid x=x x^{\sim}\right\}, \quad K=\left\{x \in S \mid x=x^{\sim} x\right\}$.

For example, if $x \in J$ then there exists $y \in S$ such that $x=y y^{\sim}$ whence, by Theorem 2(1), $x x^{\sim}=y y^{\sim}\left(y y^{\sim}\right)^{\sim}=y y^{\sim} y^{\sim \sim} y^{\sim}=y y^{\sim}=x$.

Likewise, we shall consider the subsemigroups

$S \xi=\{x \xi \mid x \in S\}, \quad \xi S=\{\xi x \mid x \in S\}$.

The subsets of idempotents of $S \xi$ and $\xi S$ are identified as follows. 
Theorem 3. $E(S \xi)=J$ and $E(\xi S)=K$.

Proof. If $j \in J$ then $j=j j^{\sim}$ whence $j=j \xi$ and therefore $J \subseteq E(S \xi)$. Conversely, if $e \in E(S \xi)$ then $e=e \xi$ gives $\xi e \xi e=\xi e$, so that $e \xi$, $\xi e \in E(S)$ and consequently $\xi \in S^{\sim} \cap I(e)=\left\{e^{\sim}\right\}$. Then $e^{\sim}=\xi$ and $e=e \xi=e e^{\sim} \in J$. Thus $E(S \xi)=J$ and dually $E(\xi S)=K$.

A further subset that is of structural importance is

$P=\left\{x \in S \mid x=x x^{\sim} x\right\}$.

Concordant with the terminology of (5), this may be called the set of perfect elements of $S$.

By the formulae in Theorem 2 , it is readily seen that, equivalently,

$P=\left\{x x^{\sim} x \mid x \in S\right\}$

with, moreover,

$(\forall x \in S) \quad\left(x x^{\sim} x\right)^{\sim}=x^{\sim}$

Since for every $j \in J$ we have $j=j j^{\sim} j$ we see that $J \subseteq P$, and similarly $K \subseteq P$. Also, for every $x \in S$, we have $x^{\sim} x^{\sim \sim} x^{\sim}=\xi x^{\sim}=x^{\sim}$ and therefore also $S^{\sim} \subseteq P$. Moreover, by the above, $S^{\sim}=P^{\sim}$ 
As seen in Example 1 above, special subgroups are in general distinct from associate subgroups. Precisely when they coincide is determined as follows.

Theorem 4. A special subgroup $S^{\sim}$ of $S$ is an associate subgroup of $S$ if and only if $P=S$.

Proof. If $P=S$ then every $x \in S$ is such that $x=x x^{\sim} x$ whence $S^{\sim} \cap A(x) \neq \emptyset$. But if $y \in$ $S^{\sim} \cap A(x)$ then $y \in S^{\sim} \cap I(x)=\left\{x^{\sim}\right\}$. Hence $S^{\sim} \cap A(x)=\left\{x^{\sim}\right\}$ and so $S^{\sim}$ is an associate subgroup. Conversely, if the special subgroup $S^{\sim}$ is an associate subgroup then for every $x \in S$ we have $x=x x^{\star} x=x x^{\sim} x \in P$ whence $P=S$.

Precisely when $P$ is a subsemigroup of $S$ is the substance of the following result.

Theorem 5. The following statements are equivalent:

(1) $P$ is a (regular) subsemigroup of $S$;

(2) $K J \subseteq P$.

Proof.

$(1) \Rightarrow(2)$ : If $P$ is a subsemigroup of $S$ then, since both $J$ and $K$ are contained in $P$, it follows that $K J \subseteq P$. 
(2) $\Rightarrow$ (1): If $x, y \in P$ then since $x^{\sim} x y y^{\sim} \in K J \subseteq P$ we have, by Theorem 2(2),

$x^{\sim} x y y^{\sim}=x^{\sim} x y y^{\sim}\left(x^{\sim} x y y^{\sim}\right)^{\sim} x^{\sim} x y y^{\sim}=x^{\sim} x y(x y)^{\sim} x y y^{\sim}$.

Pre-multiplying by $x$ and post-multiplying by $y$, we obtain $x y=x y(x y)^{\sim} x y$ so that $x y \in P$ and therefore $P$ is a subsemigroup which is regular since $S^{\sim} \subseteq P$.

In the case of an inverse transversal $S^{\circ}$ the sets which correspond to $J$ and $K$ are denoted by I and $\Lambda$. It is natural therefore to consider properties which are analogous to the principal properties listed in (4).

Recalling that $E\left(S^{\sim}\right)=\{\xi\}$, we shall say that the special subgroup $S^{\sim}$ is

prime if $K J=\{\xi\}$

weakly prime if $(K J)^{\sim}=\{\xi\}$ a quasi-ideal if $S^{\sim} S S^{\sim} \subseteq S^{\sim}$ [cf. $S^{\circ}$ multiplicative if $\Lambda \mathrm{I} \subseteq E\left(S^{\circ}\right)$ ];

[cf. $S^{\circ}$ weakly multiplicative if $\left.(\Lambda \mathrm{I})^{\circ} \subseteq E\left(S^{\circ}\right)\right]$;

[cf. $S^{\circ} S S^{\circ} \subseteq S^{\circ}$ or, equivalently, $\Lambda \mathrm{I} \subseteq S^{\circ}$ ].

In what follows we shall consider the characteristic properties of each of these types and how they are related. For this purpose, throughout what follows, $S$ will denote a regular semigroup with a special subgroup $S \sim$ whose identity element is $\xi$, and the subsets $J, K, P$ are as defined above.

\section{3. $S^{\sim}$ WEAKLY PRIME}

The weakly prime special subgroups have the following characterisations. 
Theorem 6. The following statements are equivalent:

(1) $S^{\sim}$ is weakly prime;

(2) $(\forall x, y \in S)(x y)^{\sim}=y^{\sim} x^{\sim}$;

(3) the mapping $\zeta: S \rightarrow S$ described by $\zeta: x \mapsto x^{\sim \sim}$ is a morphism;

(4) $(\forall x \in\langle E(S)\rangle) x^{\sim}=\xi$;

(5) $K J \subseteq E(P)$.

Proof.

(1) $\Rightarrow$ (2): If $S^{\sim}$ is weakly prime then, for all $x, y \in S$, we have $\left(x^{\sim} x y y^{\sim}\right)^{\sim} \in(K J)^{\sim}=\{\xi\}$. It follows by Theorem 2(2) that $(x y)^{\sim}=y^{\sim}\left(x^{\sim} x y y^{\sim}\right)^{\sim} x^{\sim}=y^{\sim} \xi x^{\sim}=y^{\sim} x^{\sim}$.

$(2) \Rightarrow(3)$ : This is clear.

(3) $\Rightarrow(2)$ : By (3) and Theorem 2(1), (xy) $=(x y)^{\sim \sim \sim}=\left(x^{\sim \sim} y^{\sim \sim}\right)^{\sim}=y^{\sim} x^{\sim}$.

$(2) \Rightarrow$ (4): If (2) holds and $e \in E(S)$ then $e^{\sim}=(e e)^{\sim}=e^{\sim} e^{\sim}$ gives $e^{\sim} \in E\left(S^{\sim}\right)$ and therefore $e^{\sim}=\xi$. An inductive argument using (2) now gives $x^{\sim}=\xi$ for every $x \in\langle E(S)\rangle$. 
(4) $\Rightarrow$ (5): If (4) holds then for all $k \in K$ and all $j \in J$ we have $(k j)^{\sim}=\xi$. Consequently, by Theorem 3,

$k j=k j \xi=k j(k j)^{\sim} \in J \subseteq E(P)$

and (5) follows.

(5) $\Rightarrow$ (1): If (5) holds then $k j \xi=k j \in E(P)$ and likewise $\xi k j \in E(P)$ whence it follows that $\xi \in S^{\sim} \cap I(k j)=\left\{(k j)^{\sim}\right\}$. Thus $(K J)^{\sim}=\{\xi\}$ and so $S^{\sim}$ is weakly prime.

Corollary. If $S^{\sim}$ is weakly prime then $J, K$ are sub-bands and $P$ is a regular subsemigroup. Also, $S^{\sim}$ is an associate subgroup of $P$ and its identity element $\xi$ is a medial idempotent of $P$.

Proof. By Theorem 2, for every $x \in J$ we have $x^{\sim}=\left(x x^{\sim}\right)^{\sim}=x^{\sim \sim} x^{\sim}=\xi$ and so $x=x x^{\sim}=x \xi$. Thus, if $x, y \in J$ then, using Theorem $6(2), x y=x y \xi=x y y^{\sim} x^{\sim}=x y(x y)^{\sim} \in J$ whence $J$, and likewise $K$, is a sub-band of $S$. That $P$ is a subsemigroup follows from Theorem 6(5) and Theorem 5. Now for every $x \in S$ we have that $x^{\sim}=\left(x x^{\sim} x\right)^{\sim}$. That $S^{\sim}=P^{\sim}$ is then an associate subgroup of $P$ follows from Theorem 4 applied to $P$. Finally, by Theorem 6(4), for every $x \in\langle E(P)\rangle$ we have $x=x x^{\sim} x=x \xi x$ and so $\xi$ is a medial idempotent of $P(3)$. 
A structure theorem for regular semigroups with an associate subgroup whose identity element is a medial idempotent (and therefore that of $P$ when $S^{\sim}$ is weakly prime) was established in (2); see also (8).

Example 3. In Example 2, $P=\{(m, n) \mid m \geqslant 0\}, J=\left\{(m, n) \mid m \geqslant 0, n_{k}=0\right\}$ and $K=\{(m, 0) \mid m \geqslant 0\}$. Then $K J=K$ and, since $K^{\sim}=\{(0,0)\}$, it follows that $S^{\sim}$ is a weakly prime special subgroup. Here $S^{\sim}$ is neither prime nor a quasi-ideal.

Example 4. If $L$ is a semilattice and $G$ is a group, consider the inverse semigroup $S=L \times G$. Here $E(S)=L \times\left\{1_{G}\right\}$ and, for every $(x, g) \in S$,

$I(x, g)=L \times\left\{g^{-1}\right\}$

For every idempotent $\xi=\left(e, 1_{G}\right)$ of $S$, the group $H_{\xi}=\{e\} \times G$ is a special subgroup of $S$ with $(x, g)^{\sim}=\left(e, g^{-1}\right)$ for every $(x, g) \in S$. With respect to this,

$P=\{(x, g) \mid x \leqslant e, g \in G\}, \quad J=K=\left\{\left(x, 1_{G}\right) \mid x \leqslant e\right\}=E(P)$.

It follows by Theorem 6 that $H_{\xi}$ is a weakly prime special subgroup of $S$. It is prime or a quasi-ideal if and only if $L$ has a bottom element 0 and $e=0$.

Example 5. Consider the semidirect product semigroup $Q \times_{\zeta} G$ of a band $Q$ with an identity element $\xi$ and a group $G$. With respect to a given morphism $\zeta: G \rightarrow$ Aut $Q$ described by $\zeta$ : $g \mapsto \zeta_{g}$, this consists of the set $Q \times G$ together with the multiplication defined by $(x, g)(y, h)=$ 
$\left(x \zeta_{g}(y), g h\right)$. That $Q \times_{\zeta} G$ is regular follows from the observation that

$(x, g)\left(\xi, g^{-1}\right)(x, g)=\left(x \zeta_{g}(\xi) \zeta_{1_{G}}(x), g g^{-1} g\right)=(x \xi x, g)=(x, g)$

Since $(x, g)(x, g)=\left(x \zeta_{g}(x), g^{2}\right)$ it follows that $E\left(Q \times_{\zeta} G\right)=\left\{\left(x, 1_{G}\right) \mid x \in Q\right\}$. Consider now the subset $H=\{\xi\} \times G$. This is clearly a subgroup of $Q \times{ }_{\zeta} G$. Moreover,

$(\xi, h) \in I(x, g) \Longleftrightarrow\left(\xi \zeta_{h}(x), h g\right),\left(x \zeta_{g}(\xi), g h\right) \in E\left(Q \times_{\zeta} G\right) \Longleftrightarrow h=g^{-1}$

so that $H \cap I(x, g)=\left\{\left(\xi, g^{-1}\right)\right\}$. Consequently, $H$ is a special subgroup of $Q \times_{\zeta} G$ under $(x, g)^{\sim}=$ $\left(\xi, g^{-1}\right)$, and $H$ is isomorphic to $G$. It follows by Theorem $6(2)$ that $H$ is weakly prime. It is readily seen that if $Q \neq\{\xi\}$ then $H$ is neither prime nor a quasi-ideal.

The situation described in Example 5 is highlighted in the following result.

Theorem 7. If $S^{\sim}$ is weakly prime then the subsemigroup $\xi P \xi$ of $P$ is isomorphic to a semidirect product of a band with an identity and a group. More precisely, if $\Delta=\left\{x \in P \mid x^{\sim}=\xi\right\}$ then $\xi \Delta \xi$ is a sub-band of $P$ with identity $\xi$. For each $g \in S^{\sim}$ the mapping $\zeta_{g}: x \mapsto g x g^{\sim}$ is an automorphism of $\xi \Delta \xi$, and $\zeta: g \mapsto \zeta_{g}$ is a morphism. Finally,

$\xi P \xi \simeq \xi \Delta \xi \times_{\zeta} S^{\sim}$

under the mapping $\vartheta: x \mapsto\left(x x^{\sim}, x^{\sim \sim}\right)$. 
Proof. If $x, y \in \Delta$ then, by Theorem 6(2), (xy) $\sim y^{\sim} x^{\sim}=\xi$ and so $\Delta$ is a subsemigroup of $P$. Now, for every $x \in \Delta, x \xi=x x^{\sim} \in J$ whence it follows that $\xi x \xi \in J \subseteq E(P)$. Also, if $x, y \in \Delta$ then $x \xi y \in \Delta$ gives $\xi x \xi \cdot \xi y \xi \in \xi \Delta \xi$ and therefore $\xi \Delta \xi$ is a sub-band of $P$ with identity element $\xi$. Moreover, for every $g \in S^{\sim}$ and every $x \in \xi \Delta \xi$, we have $\operatorname{gxg}^{\sim} \in \xi \Delta \xi$.

For each $g \in S^{\sim}$ consider the mapping $\zeta_{g}: \xi \Delta \xi \rightarrow \xi \Delta \xi$ given by $\zeta_{g}(x)=g x g^{\sim}$. We have $\zeta_{g}(\xi)=g \xi g^{\sim}=g g^{\sim}=\xi$. Moreover, if $x, y \in \xi \Delta \xi$ then

$\zeta_{g}(x y)=g x y g^{\sim}=g x \xi y g^{\sim}=g x g^{\sim} g y g^{\sim}=\zeta_{g}(x) \zeta_{g}(y)$

and so $\zeta_{g} \in$ End $\xi \Delta \xi$. Since $g^{\sim} x g \in \xi \Delta \xi$ with $\zeta_{g}\left(g^{\sim} x g\right)=g g^{\sim} x g g^{\sim}=\xi x \xi=x$, it follows that $\zeta_{g}$ is surjective. Moreover, if $\zeta_{g}(x)=\zeta_{g}(y)$ then, applying $\zeta_{g^{-1}}$ to this, we obtain $x=\xi x \xi=\xi y \xi=y$. Consequently, each $\zeta_{g} \in$ Aut $\xi \Delta \xi$.

Since $\zeta_{g}\left[\zeta_{h}(x)\right]=g h x h^{\sim} g^{\sim}=g h x(g h)^{\sim}=\zeta_{g h}(x)$, the mapping $\zeta: S^{\sim} \rightarrow$ Aut $\xi \Delta \xi$ given by $\zeta(g)=\zeta_{g}$ is a morphism.

It follows from the above that we can construct the semidirect product $\xi \Delta \xi \times_{\zeta} S^{\sim}$.

Since $x x^{\sim} \in \Delta$ by Theorem 2, it follows that $x x^{\sim} \in \xi \Delta \xi$ for every $x \in \xi P \xi$. Consider therefore the mapping $\vartheta: \xi P \xi \rightarrow \xi \Delta \xi \times{ }_{\zeta} S^{\sim}$ given by

$$
\vartheta(x)=\left(x x^{\sim}, x^{\sim \sim}\right) .
$$


That $\vartheta$ is a morphism follows from the observation that, for $x, y \in \xi P \xi$,

$$
\begin{aligned}
\vartheta(x) \vartheta(y) & =\left(x x^{\sim}, x^{\sim \sim}\right)\left(y y^{\sim}, y^{\sim \sim}\right) \\
& =\left(x x^{\sim} \zeta x^{\sim \sim}\left(y y^{\sim}\right), x^{\sim \sim} y^{\sim \sim}\right) \\
& =\left(x x^{\sim} \cdot x^{\sim \sim} y y^{\sim} x^{\sim}, x^{\sim \sim} y^{\sim \sim}\right) \\
& =\left(x \xi y y^{\sim} x^{\sim},(x y)^{\sim \sim}\right) \\
& =\left(x y(x y)^{\sim},(x y)^{\sim \sim}\right) \\
& =\vartheta(x y) .
\end{aligned}
$$

That $\vartheta$ is injective follows from the fact that if $\vartheta(x)=\vartheta(y)$ then $x x^{\sim}=y y^{\sim}$ and $x^{\sim \sim}=y^{\sim}$ whence $x=\xi x \xi=\xi x x^{\sim} x^{\sim \sim}=\xi y y^{\sim} y^{\sim \sim}=\xi y \xi=y$. Finally, given $y \in \xi \Delta \xi$ and $g \in S^{\sim}$ we have $y g \in \xi P \xi$ with

$\vartheta(y g)=\left(y g(y g)^{\sim},(y g)^{\sim \sim}\right)=\left(y g g^{\sim} y^{\sim}, y^{\sim \sim} g^{\sim \sim}\right)=(y \xi, \xi g)=(y, g)$

and so $\vartheta$ is also surjective. Consequently, $\xi P \xi \simeq \xi \Delta \xi \times_{\zeta} S^{\sim}$.

\section{4. $S^{\sim}$ A QUASI-IDEAL}

The quasi-ideal special subgroups have the following characterisations.

Theorem 8. The following statements are equivalent:

(1) $S^{\sim}$ is a quasi-ideal of $S$; 
(2) $K J \subseteq S^{\sim}$;

(3) $K J=[E(S)]^{\sim}$;

(4) $\xi P \xi=S^{\sim}$;

(5) $(\forall x \in S) \quad x^{\sim}=x^{\sim} x x^{\sim}$;

(6) $(\forall x \in S) x^{\sim \sim}=\xi x \xi$.

Proof. We establish (1) $\Rightarrow(2) \Rightarrow(3) \Rightarrow(1)$ and $(1) \Rightarrow(4) \Rightarrow(5) \Rightarrow(6) \Rightarrow(1)$.

(1) $\Rightarrow$ (2): If $S^{\sim}$ is a quasi-ideal then for all $x, y \in S$ we have $x \sim x y y^{\sim} \in S^{\sim} S S^{\sim} \subseteq S^{\sim}$ whence $K J \subseteq S^{\sim}$.

(2) $\Rightarrow$ (3): If (2) holds then for all $j \in J$ and $k \in K$ we have that $k j=(k j)^{\sim \sim} \in S^{\sim} \subseteq P$, whence it follows that $(k j)^{\sim} \in V(k j)$.

Given $j \in J$ and $k \in K$, recall (7) that the sandwich set $S(k, j)$ is given by

$S(k, j)=\{g \in E(S) \mid g=j g=g k, k g j=k j\}=j V(k j) k$.

Consider now the element $g=j(k j)^{\sim} k$ which, by the above, belongs to $S(k, j)$. Since $k j g=k g \in$ $E(S)$ and $g k j=g j \in E(S)$ we have that $k j \in S^{\sim} \cap I(g)$ whence $k j=g^{\sim}$ and consequently $K J \subseteq$ $[E(S)]^{\sim}$. Conversely, for every $e \in E(S)$ we have $e^{\sim} e e^{\sim}=e^{\sim} e \cdot e e^{\sim} \in K J \subseteq S^{\sim}$ whence, using 
Theorem 2(1), $e^{\sim}=e^{\sim \sim \sim}=\left(e^{\sim \sim} e^{\sim} e^{\sim \sim}\right)^{\sim}=\left(e^{\sim} e e^{\sim}\right)^{\sim \sim}=e^{\sim} e e^{\sim} \in K J$. Thus $[E(S)]^{\sim} \subseteq K J$ and (3) follows.

(3) $\Rightarrow$ (1): If (3) holds then $x^{\sim} x y y^{\sim} \in S^{\sim}$ whence, taking $x=\xi$, we obtain $\xi y y^{\sim} \in S^{\sim}$. It follows that $\xi y \xi=\xi y y^{\sim} y^{\sim \sim} \in S^{\sim}$ whence $x^{\sim} y z^{\sim}=x^{\sim} \xi y \xi z^{\sim} \in S^{\sim}$ and consequently $S^{\sim} S S^{\sim} \subseteq S^{\sim}$.

(1) $\Rightarrow$ (4): If (1) holds then $S^{\sim}=\xi S^{\sim} \xi \subseteq \xi S \xi \subseteq S^{\sim} S S^{\sim} \subseteq S^{\sim}$. Thus $\xi S \xi=S^{\sim} \subseteq P$ whence $\xi P \xi=\xi S \xi$ and (4) follows..

(4) $\Rightarrow$ (5): If (4) holds then for every $p \in P$ we have, by Theorem $2, \xi p \xi=(\xi p \xi)^{\sim \sim}=\xi p^{\sim \sim} \xi=$ $p^{\sim \sim}$ whence $p^{\sim} p p^{\sim}=p^{\sim} p^{\sim \sim} p^{\sim}=p^{\sim}$. Consequently,

$(\forall x \in S) \quad\left(x x^{\sim} x\right)^{\sim} x x^{\sim} x\left(x x^{\sim} x\right)^{\sim}=\left(x x^{\sim} x\right)^{\sim}$

which reduces to $x^{\sim} x x^{\sim}=x^{\sim}$ which is (5).

$(5) \Rightarrow(6)$ : If (5) holds then on pre- and post-multiplying by $x^{\sim \sim}$ we obtain (6).

(6) $\Rightarrow$ (1): If (6) holds then for all $x, y, z \in S, x^{\sim} y z^{\sim}=x^{\sim} \xi y \xi z^{\sim}=x^{\sim} y^{\sim \sim} z^{\sim} \in S^{\sim}$ whence $S^{\sim} S S^{\sim} \subseteq S^{\sim}$ and we have (1). 
Example 6. Consider the following sets of real $2 \times 2$ matrices:

$A=\left\{\left[\begin{array}{ll}x & x \\ x & x\end{array}\right],\left[\begin{array}{ll}x & 0 \\ x & 0\end{array}\right],\left[\begin{array}{cc}x & x \\ 0 & 0\end{array}\right],\left[\begin{array}{cc}x & 0 \\ 0 & 0\end{array}\right] \mid x \neq 0\right\} ; \quad B=\left\{\left[\begin{array}{ll}x & 0 \\ 0 & x\end{array}\right] \mid x \neq 0\right\}$.

Let $S=A \cup B$. Then $S$ is a regular monoid with

$E(S)=\left\{\left[\begin{array}{cc}\frac{1}{2} & \frac{1}{2} \\ \frac{1}{2} & \frac{1}{2}\end{array}\right],\left[\begin{array}{ll}1 & 1 \\ 0 & 0\end{array}\right],\left[\begin{array}{ll}1 & 0 \\ 1 & 0\end{array}\right],\left[\begin{array}{ll}1 & 0 \\ 0 & 0\end{array}\right],\left[\begin{array}{ll}1 & 0 \\ 0 & 1\end{array}\right]\right\}$

For each $X \in S$ define $X^{\sim}=\left[\begin{array}{cc}x^{-1} & 0 \\ 0 & 0\end{array}\right]$. Then $S^{\sim}=\left\{\left[\begin{array}{ll}x & 0 \\ 0 & 0\end{array}\right] \mid x \neq 0\right\}$ is a special subgroup. Here

$K=\left\{\left[\begin{array}{ll}1 & 1 \\ 0 & 0\end{array}\right],\left[\begin{array}{ll}1 & 0 \\ 0 & 0\end{array}\right]\right\} ; \quad J=\left\{\left[\begin{array}{ll}1 & 0 \\ 1 & 0\end{array}\right],\left[\begin{array}{ll}1 & 0 \\ 0 & 0\end{array}\right]\right\}$

and consequently

$K J=\left\{\left[\begin{array}{ll}2 & 0 \\ 0 & 0\end{array}\right],\left[\begin{array}{ll}1 & 0 \\ 0 & 0\end{array}\right]\right\}=[E(S)]^{\sim}$

Thus, by Theorem 8 , the special subgroup $S^{\sim}$ is a quasi-ideal of $S$.

Clearly, $S^{\sim}$ is neither prime nor weakly prime.

When $S^{\sim}$ is a quasi-ideal, we have the following useful identification of the subsemigroups $\xi S$ and $S \xi$ as, respectively, the $\mathcal{R}$-class and the $\mathcal{L}$-class of $\xi$.

Theorem 9. If $S^{\sim}$ is a quasi-ideal then 
(1) $\xi S=R_{\xi}=\{x \in S \mid x \mathcal{R} \xi\}, \quad S \xi=L_{\xi}=\{x \in S \mid x \mathcal{L} \xi\}$;

(2) $J=E\left(L_{\xi}\right)$ and is a left zero semigroup;

(3) $K=E\left(R_{\xi}\right)$ and is a right zero semigroup;

(4) $J \cap K=\{\xi\}$.

Proof.

(1) If $x \mathcal{L} \xi$ then $S x=S \xi$ whence there exists $y \in S$ such that $x=y \xi$. Then $x=x \xi \in S \xi$. Conversely, if $x \in S \xi$ then $x=x \xi$ gives, on the one hand $S x \subseteq S \xi$, and on the other by Theorem 8(5), $x^{\sim} x=x^{\sim} x \xi=x^{\sim} \xi x \xi=x^{\sim} x^{\sim \sim}=\xi$, whence $S \xi \subseteq S x$. The resulting equality now gives $x \mathcal{L} \xi$. Thus $S \xi=L_{\xi}$ and similarly $\xi S=R_{\xi}$.

(2) and (3) follow immediately from (1) and Theorem 3.

As for (4), it follows from (2) and (3) that

$J \cap K=E\left(L_{\xi}\right) \cap E\left(R_{\xi}\right)=E\left(L_{\xi} \cap R_{\xi}\right)=E\left(H_{\xi}\right)=\{\xi\}$.

Theorem 10. If $S^{\sim}$ is a quasi-ideal then $P$ is an ideal of $S$, and $S^{\sim}$ is a group transversal of $P$. 
Proof. If $S^{\sim}$ is a quasi-ideal then, from $S^{\sim} \subseteq P$ and Theorem 8(2), we obtain $K J \subseteq P$. It follows by Theorem 5 that $P$ is a regular subsemigroup of $S$. Moreover, if $p \in P$ and $x \in S$ then, by Theorem 9(2), $p x(p x)^{\sim} p x=p p^{\sim} p x(p x)^{\sim} p x=p p^{\sim} p x=p x$ whence $P S \subseteq P$. Similarly, by Theorem 9(3), $S P \subseteq P$ and consequently $P$ is an ideal of $S$.

Now since Theorem 8(5) holds, if $x \in P$ then $x^{\sim} \in V(x)$ so $S^{\sim} \cap V(x) \neq \emptyset$. But if $y \in S^{\sim} \cap V(x)$ then $y \in S^{\sim} \cap I(x)=\left\{x^{\sim}\right\}$. Hence $\left|S^{\sim} \cap V(x)\right|=1$ and so $S^{\sim}$ is a group transversal of $P$ with $x^{\circ}=x^{\sim}$

Corollary. If $S^{\sim}$ is a quasi-ideal then $P$ is completely simple.

Proof. Since $S^{\sim}$ is a group transversal of $P$, this follows from a basic theorem of Saito (9); see also (1, Theorem 4.3).

When $S^{\sim}$ is a quasi-ideal, the structure of $P$ can be described in terms of $S \xi=L \xi$ and $\xi S=R \xi$. For this purpose, consider the spined product set

$S \xi|\times| \xi S=\left\{(x, a) \in S \xi \times \xi S \mid x^{\sim}=a^{\sim}\right\}$. 
For $x, y \in S \xi$ and $a, b \in \xi S$, it follows by Theorem 2 and Theorem 8(5) that

$\left(x x^{\sim} a y\right)^{\sim}=\left(x^{\sim} x x^{\sim} a y\right)^{\sim} x^{\sim}=\left(x^{\sim} a y\right)^{\sim} x^{\sim}=(a y)^{\sim} x^{\sim \sim} x^{\sim}=(a y)^{\sim}$.

Similarly, $\left(a y b^{\sim} b\right)^{\sim}=(a y)^{\sim}$. We can therefore define a law of composition on $S \xi|\times| \xi S$ by the prescription

$(x, a)(y, b)=\left(x x^{\sim} a y, a y b^{\sim} b\right)$.

Then

$$
\begin{aligned}
& {[(x, a)(y, b)](z, c)=\left(x x^{\sim} a y, a y b^{\sim} b\right)(z, c)} \\
& =\left(x x^{\sim} a y\left(x x^{\sim} a y\right)^{\sim} a y b^{\sim} b z, a y b^{\sim} b z c \sim c\right) \\
& =\left(x x^{\sim} a y\left(x^{\sim} x x^{\sim} a y\right)^{\sim} x^{\sim} a y b \sim b z, a y b^{\sim} b z c^{\sim} c\right) \quad \text { by Theorem } 2 \\
& =\left(x x^{\sim} a y \cdot\left(x^{\sim} a y\right)^{\sim} x^{\sim} a y \cdot b^{\sim} b \cdot z, a y b^{\sim} b z c^{\sim} c\right) \quad \text { by Theorem 8(5) } \\
& =\left(x x^{\sim} a y b^{\sim} b z, a y b^{\sim} b z c^{\sim} c\right) ; \quad \text { by Theorem 9(3) } \\
& (x, a)[(y, b)(z, c)]=(x, a)\left(y y^{\sim} b z, b z c^{\sim} c\right) \\
& =\left(x x \sim a y y^{\sim} b z, a y y^{\sim} b z(b z c \sim c) \sim b z c \sim c\right) \\
& =\left(x x^{\sim} a y y^{\sim} b z, a y y^{\sim} b z c^{\sim}\left(b z c^{\sim} c c^{\sim}\right)^{\sim} b z c^{\sim} c\right) \quad \text { by Theorem } 2 \\
& =\left(x x^{\sim} a y y^{\sim} b z, a \cdot y y^{\sim} \cdot b z c^{\sim}\left(b z c^{\sim}\right)^{\sim} \cdot b z c^{\sim} c\right) \quad \text { by Theorem 8(5) } \\
& =\left(x x^{\sim} a y y^{\sim} b z, a y y^{\sim} b z c^{\sim} c\right) . \quad \text { by Theorem 9(2) }
\end{aligned}
$$


Since $y^{\sim}=b^{\sim}$, these products are equal and therefore $S \xi|\times| \xi S$ is a semigroup which we now show is isomorphic to $P$.

Theorem 11. If $S^{\sim}$ is a quasi-ideal then $P=J S^{\sim} K$ and $P \simeq S \xi|\times| \xi S=L_{\xi}|\times| R_{\xi}$.

Proof. For every $x \in P$ we have $x=x x^{\sim} x=x x^{\sim} x^{\sim \sim} x^{\sim} x \in J S^{\sim} K$ so that $P \subseteq J S^{\sim} K$. The reverse inclusion is immediate from the fact that $J, S, K \subseteq P$ and $P$ is a subsemigroup.

Consider now the mapping $\vartheta: P \rightarrow S \xi|\times| \xi S$ given by $\vartheta(p)=(p \xi, \xi p)$.

(a) $\vartheta$ is injective.

If $\vartheta(p)=\vartheta(q)$ then, by Theorem 2(1),

$p=p p^{\sim} p=p \xi(p \xi)^{\sim} \xi p=q \xi(q \xi)^{\sim} \xi q=q q^{\sim} q=q$.

(b) $\vartheta$ is surjective. Let $(p, q) \in S \xi|\times| \xi S$. Then $p^{\sim}=q^{\sim}$ and, by Theorem $8(5)$,

$\vartheta\left(p p^{\sim} q\right)=\left(p p^{\sim} q \xi, \xi p p^{\sim} q\right)=\left(p p^{\sim} q^{\sim \sim}, p^{\sim \sim} p^{\sim} q\right)=(p \xi, \xi q)=(p, q)$.

(c) $\vartheta$ is a morphism. 
In fact,

$$
\begin{aligned}
\vartheta(p) \vartheta(q)=(p \xi, \xi p)(q \xi, \xi q) & =\left(p \xi(p \xi)^{\sim} \xi p q \xi, \xi p q \xi(\xi q)^{\sim} \xi q\right) \\
& =\left(p p^{\sim} p q \xi, \xi p q q^{\sim} q\right) \\
& =(p q \xi, \xi p q) \\
& =\vartheta(p q) .
\end{aligned}
$$

It follows by $(a),(b),(c)$ that $\vartheta$ is a semigroup isomorphism.

Example 7. In Example 6 we have $\xi=\left[\begin{array}{ll}1 & 0 \\ 0 & 0\end{array}\right]$ and

$S \xi=\left\{\left[\begin{array}{ll}x & 0 \\ x & 0\end{array}\right],\left[\begin{array}{ll}x & 0 \\ 0 & 0\end{array}\right] \mid x \neq 0\right\}, \quad \xi S=\left\{\left[\begin{array}{cc}x & x \\ 0 & 0\end{array}\right],\left[\begin{array}{cc}x & 0 \\ 0 & 0\end{array}\right] \mid x \neq 0\right\}$.

In this example, as can readily be verified, $P$ coincides with the subsemigroup $A$ and Theorems 10 and 11 apply.

\section{5. $S^{\sim}$ PRIME}

Finally, we consider the special subgroups that are prime. In relation to the previous properties, these have the following characterisation. 
Theorem 12. The following statements are equivalent:

(1) $S^{\sim}$ is prime;

(2) $S^{\sim}$ is weakly prime and a quasi-ideal of $S$;

(3) $S^{\sim}$ is a quasi-ideal of $S$ and $\xi$ is a middle unit of $P$.

Proof.

(1) $\Rightarrow$ (2): If (1) holds then $K J=\{\xi\}$ and consequently, by Theorem 6(5) and Theorem 8(2), $S^{\sim}$ is both weakly prime and a quasi-ideal.

(2) $\Rightarrow(3)$ : If $S^{\sim}$ is weakly prime and a quasi-ideal then, by Theorem 8(5) and Theorem 6(4), for all $x, y \in P$,

$x y=x \xi x^{\sim} x y y^{\sim} \xi y=x\left(x^{\sim} x y y^{\sim}\right) \sim \sim y=x \xi y$

whence $\xi$ is a middle unit of $P$.

(3) $\Rightarrow(1)$ : If $S^{\sim}$ is a quasi-ideal and $\xi$ is a middle unit of $P$ then, by Theorem $9(2,3)$,

$x^{\sim} x y y^{\sim}=x^{\sim} x \xi y y^{\sim}=x^{\sim} x \xi=\xi$. 
Consequently $K J=\{\xi\}$ and so $S^{\sim}$ is prime.

Theorem 13. If $S^{\sim}$ is prime then

(1)JK $=E(P)=\left\{x \in P \mid x^{\sim}=\xi\right\}$

(2)P is orthodox.

Proof.

(1) If $x=j k \in J K$ then, since $J, K \subseteq P$ and, by Theorem 12 and the Corollary to Theorem 6,P is a subsemigroup, we see that $x \in P$. Moreover, by Theorem 9, $x \xi=j k \xi=j \xi=j$ and $\xi x=\xi j k=$ $\xi k=k$. Consequently, since $\xi$ is a middle unit of $P$ by Theorem 12, $x=j k=x \xi \xi x=x^{2}$. Thus $J K \subseteq E(P)$.

Conversely, if $e \in E(P)$ then, by Theorem 6(4) and Theorem 9,

$e=e e^{\sim} e=e \xi e=e \xi \xi e \in J K$

whence $E(P) \subseteq J K$ and we have equality.

If now $x \in P$ with $x^{\sim}=\xi$ then $x=x x^{\sim} x=x \xi x=x^{2}$, so $\left\{x \in P \mid x^{\sim}=\xi\right\} \subseteq E(P)$. The reverse inclusion follows by Theorem 5(4). 
(2) If $e, f \in E(P)$ then, by (1) and the fact that $\xi$ is a middle unit of $P$,

ef $\in J K J K=J \xi K=J K=E(P)$

and so $P$ is orthodox.

If $S^{\sim}$ is prime then, by the Corollary to Theorem $10, P$ is a completely simple subsemigroup which, by Theorem 13, is orthodox. In this case, $P$ can therefore be expressed as the cartesian product of a group and a rectangular band. The identification of these is the substance of the following.

Theorem 14. If $S^{\sim}$ is prime then $J \times K$ is a rectangular band and $P \simeq J \times S^{\sim} \times K$.

Proof. That $J \times K$ is a rectangular band is immediate from Theorem $9(2,3)$.

Consider the mapping $\vartheta: P \rightarrow J \times S^{\sim} \times K$ given by $\vartheta(x)=\left(x x^{\sim}, x^{\sim \sim}, x^{\sim} x\right)$.

Since every $x \in P$ is such that $x=x x^{\sim} x=x x^{\sim} \cdot x^{\sim \sim} \cdot x^{\sim} x$, it is clear that $\vartheta$ is injective.

To see that $\vartheta$ is also surjective, let $(j, g, k) \in J \times S^{\sim} \times K$ and consider the element $j g k$ which, by Theorem 11, belongs to $P$. By Theorem $9(2), j g k(j g k)^{\sim}=j j^{\sim} \cdot j g k(j g k)^{\sim}=j j^{\sim}=j$, and likewise, by Theorem 8(3), $(j g k)^{\sim} j g k=k$. Since, by Theorem 5, $(j g k)^{\sim \sim}=g^{\sim \sim}=g$ it follows that $\vartheta(j g k)=(j, g, k)$ and hence $\vartheta$ is surjective. 
Furthermore, for all $x, y \in P$, it follows by Theorem 9 and Theorem 6 that

$$
\begin{aligned}
\vartheta(x) \vartheta(y) & =\left(x x^{\sim}, x^{\sim \sim}, x^{\sim} x\right)\left(y y^{\sim}, y^{\sim \sim}, y^{\sim} y\right) \\
& =\left(x x^{\sim} y y^{\sim}, x^{\sim \sim} y^{\sim}, x^{\sim} x y^{\sim} y\right) \\
& =\left(x x^{\sim},(x y)^{\sim \sim}, y^{\sim} y\right) \\
& =\left(x x^{\sim} \cdot x y(x y)^{\sim},(x y)^{\sim \sim},(x y)^{\sim} x y \cdot y^{\sim} y\right) \\
& =\left(x y(x y)^{\sim},(x y)^{\sim \sim},(x y)^{\sim} x y\right) \\
& =\vartheta(x y) .
\end{aligned}
$$

Consequently, $\vartheta$ is a semigroup isomorphism.

Example 8. Consider the subsemigroup $T=-\mathbb{N} \times \mathbb{Z}$ of the semigroup $S$ in Example 2. Here $T^{\sim}=\left\{\left(0, n_{k}\right) \mid n \in \mathbb{Z}\right\}$ with $J=\left\{(0, n) \mid n_{k}=0\right\}$ and $K=\{(0,0)\}$. Then $K J=\{(0,0)\}$ and so $T^{\sim}$ is prime. Here $P=\{(0, n) \mid n \in \mathbb{Z}\}$ and, since $K$ is trivial, $P \simeq J \times T^{\sim}$.

Example 9. Similar to Example 4, let $S=L \times G$ where $L$ is a left zero semigroup and $G$ is a group. Here $E(S)=L \times\left\{1_{G}\right\}$ and $I(x, g)=L \times\left\{g^{-1}\right\}$. For every idempotent $\xi=\left(e, 1_{G}\right)$ the group $H_{\xi}=\{e\} \times G$ is a special subgroup with $(x, g)^{\sim}=\left(e, g^{-1}\right)$. Here $(x, g)(x, g)^{\sim}=\left(x, 1_{G}\right)$ and $(x, g)^{\sim}(x, g)=\left(e, 1_{G}\right)$ so that $J=E(S)$ and $K=\{\xi\}$. Then $K J=\{\xi\}$ and so every $H_{\xi}$ is prime. 


\section{FUNDING}

This work was partially supported by the Portuguese Foundation for Science and Technology through the grant UID/MAT/00297/2013 (CMA).

\section{REFERENCES}

1. Blyth, T. S. (2000). Inverse transversals-A guided tour. Proceedings of an International Conference on Semigroups, Braga, 1999. World Scientific.

2. Blyth, T. S., Martins, P. M. (1997). On associate subgroups of regular semigroups. Communications in Algebra 25:2147-2156.

3. Blyth, T. S., McFadden, R. (1983). On the construction of a class of regular semigroups. Journal of Algebra 81:1-22.

4. Blyth, T. S., Almeida Santos, M. H. (2001). A classification of inverse transversals. Communications in Algebra 29:611-624.

5. Blyth, T. S., Almeida Santos, M. H. (2015). E-special ordered regular semigroups. Communications in Algebra 43:3294-3312.

6. Croisot, R. (1953). Demi-groupes inversifs et demi-groupes réunions de demi-groupes simples. Ann. Sci. École Norm. Sup. 70:361-379.

7. Higgins, P. M. (1992). Techniques of Semigroup Theory. Oxford: Oxford University Press.

8. Petrich, M. (2011). On some medial semigroups with an associate subgroup. Semigroup Forum 83:412-446.

9. Saito, T. (1987). Quasi-orthodox semigroups with inverse transversals. Semigroup Forum 36:4754. 